\title{
Perpendicular and turbulent flow in acute aortic paravalvular leak: case report
}

\author{
Hongyu Zhou, Hongyang Chen, Lingcan Tan, Yu Li \\ Department of Anesthesiology, West China Hospital, Sichuan University, Chengdu, China \\ Correspondence to: Yu Li. Department of Anesthesiology, West China Hospital, Sichuan University, No.37 Guoxue Street, Chengdu 610041, China. \\ Email: biaojieli@163.com.
}

\begin{abstract}
Paravalvular leaks (PVL) are well-recognized complications after artificial valve replacement and symptomatic periprosthetic leaks are closely associated with increased mobility and mortality. Owing to the difference in types of prosthetic valve and options of suture technique, echocardiography of PVL can be diverse and challenge to diagnose. Herein, we report a patient with continuous low cardiac output syndrome on the second day after surgery after aortic replacement, which was misdiagnosed as coronary-left ventricular fistula by transthoracic echocardiogram (TTE). However, transesophageal echocardiogram (TEE) detected a perpendicular and turbulent jet flow from the outer side of the sewing ring, which was eventually diagnosed as an acute aortic PVL combined with cardiac angiography. The significant PVLs were treated by reoperation previously, while they are recommended for transcatheter closure recently. This patient was received successful percutaneous closure by the Cardi-O-Fix Plug. The cardiac symptom was relieved without additional complications. This report highlights the important role of multi-modality imaging, including the combination of TTE, TEE and cardiac angiography, in the diagnosis and treatment of PVL. Percutaneous closure is an appropriate option for the repair of PVL with long-term symptom relief and reduced mortality compared to reoperation. Therefore, this article describes the diagnosis and treatment of this patient with acute aortic PVL.
\end{abstract}

Keywords: Aortic paravalvular leak (aortic PVL); transesophageal echocardiography; transcatheter closure; case report

Submitted Mar 24, 2021. Accepted for publication Jun 25, 2021.

doi: 10.21037/apm-21-698

View this article at: https://dx.doi.org/10.21037/apm-21-698

\section{Introduction}

Aortic paravalvular leak (PVL), an abnormal retrograde flow between the sewing ring and native annulus, is a common complication following aortic valve replacement (AVR). According to the current report, the patients with symptomatic PVL have increased mortality (1). Transesophageal echocardiogram (TEE) plays a significant role in assessing the location and severity of PVL. We report an older man diagnosed as PVL by TEE, and ultimately received successful interventional transcatheter closure. We present the following case in accordance with the CARE reporting checklist (available at https://dx.doi. org/10.21037/apm-21-698).

\section{Case presentation}

A 72-year-old man was referred to our emergency room due to exertional dyspnea for 3 years, aggravation for 1 week. He had 3 years history of valvular heart disease, and home medication included bisoprolol. Abnormal elevated cardiac enzymes, including troponin level trending up to $52.5(0-14 \mathrm{ng} / \mathrm{L})$ and brain natriuretic peptide up to 4,321 (8-21 mg/dL), suggested the presence of congestive heart failure. Preoperative transthoracic echocardiography (TTE) of the patient showed calcification of the aortic valve. TTE had proven severe aortic valve regurgitation and mild aortic valve stenosis, with significantly dilated left ventricular of $81 \mathrm{~mm}$, and impaired left ventricular eject 


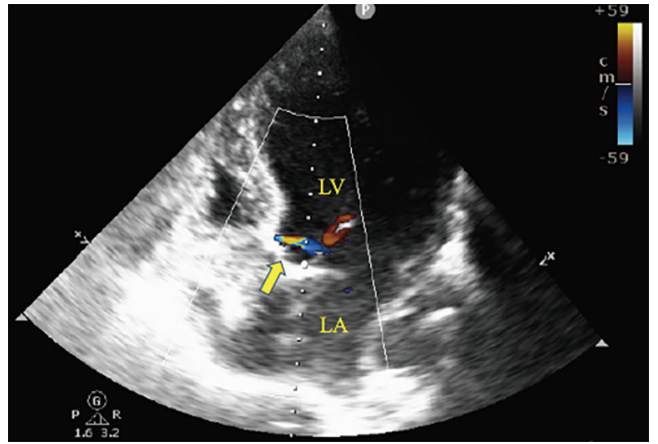

Figure 1 TTE in apical 5-chamber views with color Doppler. The arrow shows abnormal blood flow in the LVOT, suspecting coronary-left ventricular fistula. LV, left ventricle; LA, left atrium; TTE, transthoracic echocardiogram; LVOT, left ventricular outflow tract.

fraction of $39 \%$. Then the patient was admitted to the cardiovascular surgery unit. On admission, initial vital signs showed blood pressure of $111 / 75 \mathrm{mmHg}$, respiration rate of 20 breaths/min, and heart rate of 68 beats per min. One week after admission, the patient received AVR with a biological valve under cardiopulmonary bypass. No abnormal movement or blood flow of the valve was noticed in TEE. The patient was routinely transferred into the intensive care unit postoperatively.

The second day after surgery, the patient showed continuous low cardiac output syndrome, even with high dose cardiotonic. Postoperative TTE revealed dilated left ventricular of $73 \mathrm{~mm}$, and a significantly reduced eject fraction of $30 \%$ by ultrasound doctor. Whereas, in the apical 5-chamber view, TTE detected dubious flow in the left ventricular outflow tract (LVOT), which suspected coronary-left ventricular fistula (Figure 1), without abnormal ventricular wall motion. ECG showed atrial fibrillation without ST-segment depression, which was not consistent with the clinical manifestations of coronary-left ventricular fistula. Therefore, the patient was examined by TEE to confirm the diagnosis. In the long-axis view of the aortic valve, color Doppler observed a turbulent flow perpendicularly from the outer side of the sewing ring, with a high velocity of $4.52 \mathrm{~m} / \mathrm{s}$ (Figure $2 A, 2 B$ ). Furthermore, short-axis view also revealed a considerable flow convergence originating from outer side of the sewing ring and adjacent to the stent post (Figure 2C,2D). These TEE findings suggested that PVL was highly suspectable, then subsequent ascending aortic angiography confirmed it (Figure 3). PVL diameter near the aortic root was approximate $3 \mathrm{~mm}$ measured by angiography. Afterward, the leak was passed by a hydrophilic Terumo 0.32-inch guidewire and a $5 \mathrm{~F}$ delivery catheter was placed into the left ventricle. Transcatheter occlusion was successfully made to intervene by performing a $10 \mathrm{~mm}$ Vascular Plug device (Starway Medical Technology Inc., Cardi-O-Fix Plug Occluder, Beijing, China) (Figure 4A). After the device was placed, aortic root angiography revealed no PVL and coronary obstruction (Figure 4B). Subsequently, TTE was reexamined 8 days after closure of PVL, showed reduced left ventricular of $50 \mathrm{~mm}$, left ventricular motion synchrony, slightly increased EF of $38 \%$, and no observation of PVL (Figure 5). The patient was discharged from the hospital satisfactorily after about 4 weeks. No signs of recurrence have been detected in 2 months of follow-up.

All procedures performed in studies involving human participants were in accordance with the ethical standards of the institutional and/or national research committee(s) and with the Helsinki Declaration (as revised in 2013). Signed written informed consent from the patient was obtained for reporting this case.

\section{Discussion}

PVL has been demonstrated $2-10 \%$ in aortic prosthetic implantation and $7-17 \%$ in mitral position postoperatively (2). While most patients with PVL are asymptomatic, approximately $1-5 \%$ of PVL patients develop significant clinical consequences with higher mortality, such as severe heart failure and hemolytic anemia (3). Despite experiences and guidelines for evaluating and managing PVL, the diagnosis and differential diagnosis of PVL can be as difficult as in this case.

In our case, the patient was once misdiagnosed as coronary artery-left ventricular fistula. Coronary artery fistula is an abnormal connection between coronary artery and cardiac chamber or major vessels, accounts for $0.2-0.4 \%$ of congenital heart disease (4). The majority of coronary fistula opens to the right heart, very rare in the left ventricular. 2D echocardiography shows dilated and tortuous coronary artery and associated enlarged feeding artery, sometimes appearing the origin or course of the fistula vessel (5-7). Color Doppler helps identify the entrance and end site of drainage, characterized by a continuous and slow shunt both in systolic and diastolic periods in the coronary artery fistula (5). PVL usually appears as high velocity, abnormal jet flow originating from outer side prosthetic valve frame without the drainage 

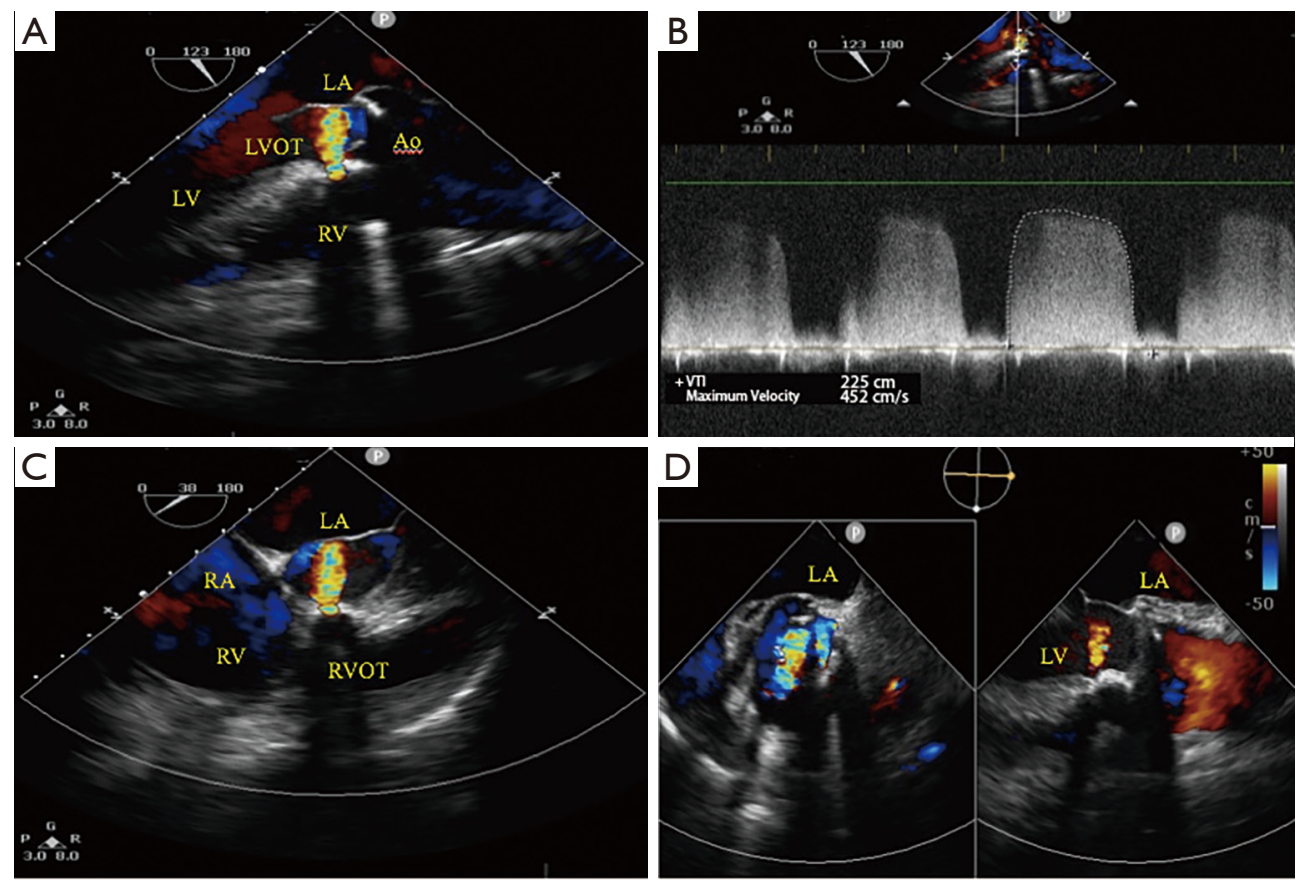

Figure 2 TEE revealing prosthetic aortic valve regurgitation outside the edge of sewing ring both from long axis and short axis view. (A) In the long-axis view of the aortic valve, a turbulent flow perpendicularly from the outer side of the sewing ring. (B) In the long-axis view of the aortic valve, the flow with high velocity of $4.52 \mathrm{~m} / \mathrm{s}$. (C) In the short-axis view of the aortic valve, a considerable flow convergence originating from outer side of the sewing ring and adjacent to the stent post. (D) The abnormal flow from the outer side of the sewing ring in biplane TEE, the left side shows the short-axis view of the aortic valve, the right side shows the automatically generated long-axis view of the aortic valve. LA, left atrium; LV, left ventricle; RA, right atrium; RV, right ventricle; LVOT, left ventricular outflow tract; RVOT, right ventricular outflow tract; Ao, aorta; TEE, transesophageal echocardiogram.

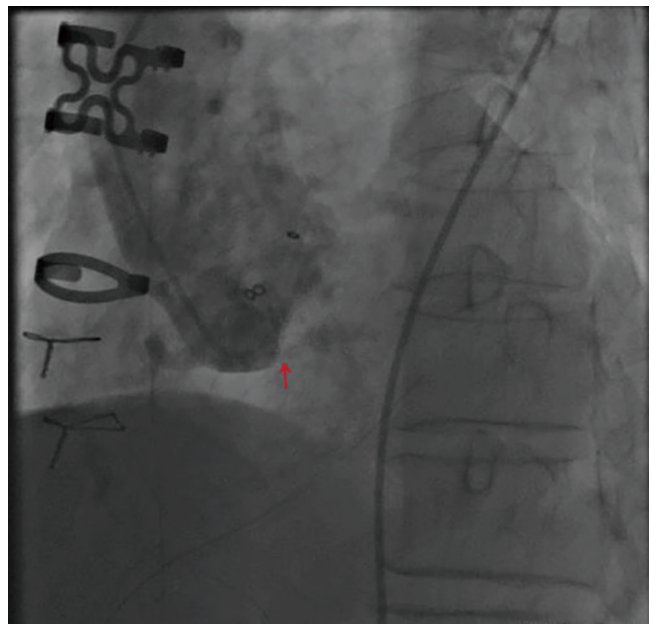

Figure 3 Aortic root angiography confirming PVL. The arrow shows the aortic PVL. PVL, paravalvular leak. process. However, due to the difference in prosthetic valve and suture technique, color Doppler of PVL can be diverse and sometimes challenging to distinguish. Perpendicular jet flow can be observed in transvalvular leakage after AVR (8). However, PVL has rarely been reported as unusual perpendicular and turbulent flow in cases of AVR with stented biological valves (9), which is consistent with our case. Due to the limitation of acoustic shadow, the aortic prosthesis cannot be displayed clearly, resulting in the misidentification of abnormal color Doppler signals and the underestimation of the severity of regurgitation. Compared with TTE, TEE can display heart structure more clearly in real-time, which is helpful for the quantitative evaluation of heart function and heart structure. In addition, multimodality imaging, including the combination of TTE, TEE and cardiac angiography, may be needed to confirm 

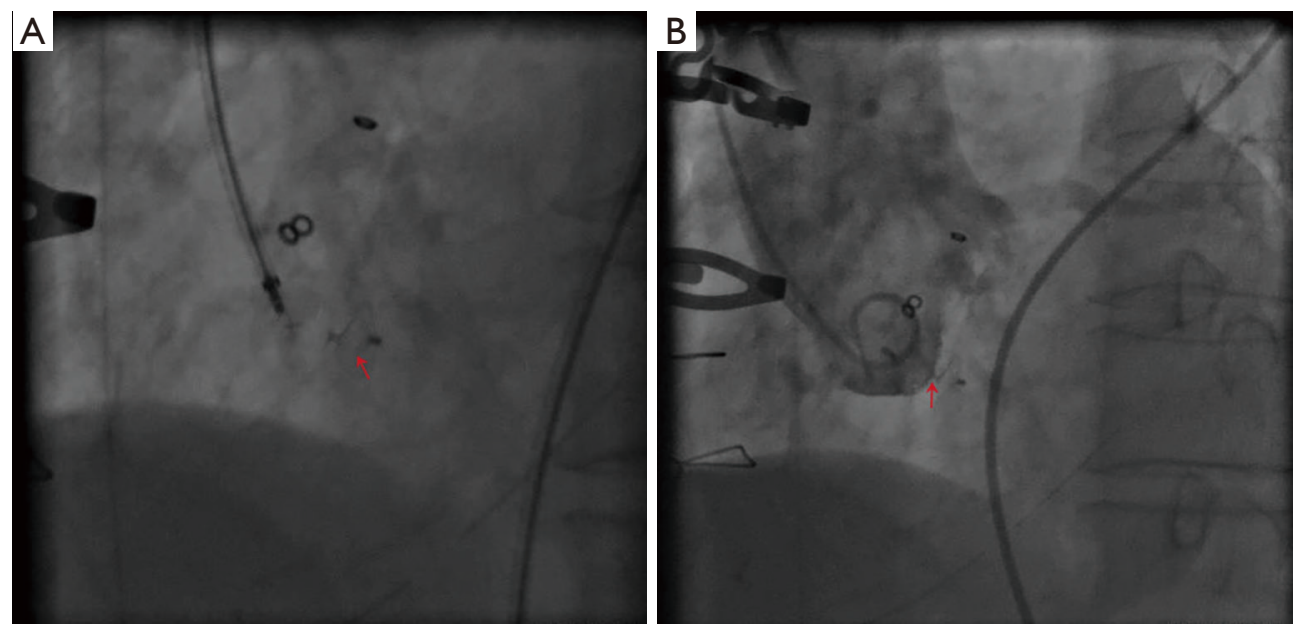

Figure 4 The PVL was successfully closed by the transcatheter occlusion. (A) Releasing of Vascular Plug device after positioning PVL. The arrow shows the Cardi-O-Fix Plug. (B) Aortic root angiography excludes PVL after transcatheter closure. The arrow shows no PVL. PVL, paravalvular leak.

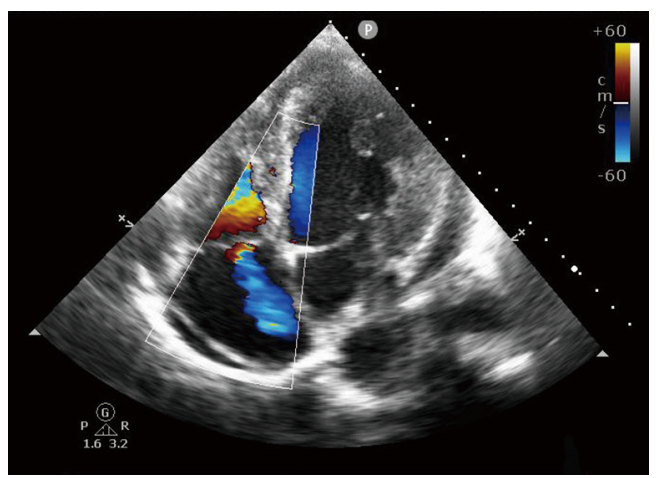

Figure 5 TTE in apical 5-chamber views with color Doppler 8 days after closure of PVL, showing no observation of PVL. TTE, transthoracic echocardiogram; PVL, paravalvular leak.

and assess abnormal blood flow considering the complexity of diagnosis $(2,3)$. As in this case, the possibility of PVL was suspected in TEE, and subsequently confirmed by cardiac angiography, highlighting the use of multi-modality diagnosis in PVL.

As Matteucci reported, early occurrence of PVL is mainly related to technical risk factors, whereas late occurrence is related to infection (10). In this case, the PVL occurred on the second day after surgery. There was no evidence of infective endocarditis after surgery. As previous studies reported, the main factors of PVL are older age, smaller body surface area (BSA), female sex, operating surgeon, endocarditis, severe calcification of the annulus, bioprosthetic valve implantation (10,11). PVL was more common in the supra-annular aortic valves used in this patient than in an intra-annular position (12).

In this case, the patient developed severe PVL in the immediate postsurgical period, making it difficult to determine which treatment is beneficial for the patient. Studies have shown that most mild PVLs were remission or remain unchanged with no increased mortality during long-time follow-up (11). Reoperation has been a traditional treatment for significant PVL, but it is associated with high mortality. In the recent two decades, transcatheter closure has emerged as the available therapy for patients with PVL, and is especially recommended for those patients with significant symptoms at high surgical risk (2). Although it has been reported that percutaneous closure of leaks is not advised in the immediate postsurgical period (13), many literatures have shown that a successful percutaneous aortic PVL closure can alleviate cardiac symptoms and reduce mortality (14). Patients treated with percutaneous closure had fewer complications after surgery (15). As reported, reduction in percutaneous aortic PVL is associated with long-term symptom relief and lower rates of reoperation. The benefit is greatest when PVL decreased to a mild grade (14).

Despite that no device has been approved for PVL occlusion by FDA, available devices include Occlutech PLD (16) and Amplatzer vascular plugs (AVP II and III) $(17,18)$ have been demonstrated to achieve successful PVL closure in the majority of cases. The Cardi-O-Fix Plug used in this case, is a plugging device made in China. 
The occluder is safe and effective for the treatment of patient as compared to the Amplatzer septal occluder in the percutaneous closure of atrial septal defect (19), and patent ductus arteriosus (20). On the other hand, successful closure requires experienced interventional cardiologists to be familiar with complex catheter techniques and have the facility to perform them. The success of these procedures is not only dependent on operator skills but also on the quality of the imaging.

This paper reports a successful case of repair of aortic paravalvular leakage with a domestic embolization device. The accurate diagnosis with TEE and angiography can avoid secondary thoracotomy, reduce hospitalization costs and shorten the length of ICU stay.

\section{Conclusions}

The PVL is a common complication after AVR. In this case, PVL was showed as unusual perpendicular and turbulent flow and difficult to distinguish from coronary artery fistula. TEE plays a significant role in the diagnosis of PVL and multi-modality imaging should be advocated. Percutaneous closure had been shown as an appropriate option for the repair of PVL.

\section{Acknowledgments}

Funding: None.

\section{Footnote}

Reporting Checklist: The authors have completed the CARE reporting checklist. Available at https://dx.doi. org/10.21037/apm-21-698

Peer Review File: Available at https://dx.doi.org/10.21037/ apm-21-698

Conflicts of Interest: All authors have completed the ICMJE uniform disclosure form (available at https://dx.doi. org/10.21037/apm-21-698). The authors have no conflicts of interest to declare.

Ethical Statement: The authors are accountable for all aspects of the work in ensuring that questions related to the accuracy or integrity of any part of the work are appropriately investigated and resolved. All procedures performed in studies involving human participants were in accordance with the ethical standards of the institutional and/or national research committee(s) and with the Helsinki Declaration (as revised in 2013). Written informed consent was obtained from the patient.

Open Access Statement: This is an Open Access article distributed in accordance with the Creative Commons Attribution-NonCommercial-NoDerivs 4.0 International License (CC BY-NC-ND 4.0), which permits the noncommercial replication and distribution of the article with the strict proviso that no changes or edits are made and the original work is properly cited (including links to both the formal publication through the relevant DOI and the license). See: https://creativecommons.org/licenses/by-nc-nd/4.0/.

\section{References}

1. 1. Ruiz CE, Mathur AP. Paravalvular Leak Closure: Time to Standardize Clinical Endpoints? JACC Cardiovasc Interv 2016;9:2427-8.

2. Kliger C, Eiros R, Isasti G, et al. Review of surgical prosthetic paravalvular leaks: diagnosis and catheter-based closure. Eur Heart J 2013;34:638-49.

3. Bernard S, Yucel E. Paravalvular Leaks-From Diagnosis to Management. Curr Treat Options Cardiovasc Med 2019;21:67.

4. Said SA, Lam J, van der Werf T. Solitary coronary artery fistulas: a congenital anomaly in children and adults. A contemporary review. Congenit Heart Dis 2006;1:63-76.

5. Kumar B, Kumar A, Kumar G, et al. Role of transesophageal echocardiography in surgical retrieval of embolized amplatzer device and closure of coronarycameral fistula. Ann Card Anaesth 2017;20:351-4.

6. Chung PC, Huang PC, Liu YF, et al. Transesophageal echocardiography, more than a diagnostic tool: use during surgical ligation of coronary artery fistulae - a case report. J Cardiothorac Surg 2012;7:28.

7. Bittencourt MS, Seltman M, Achenbach S, et al. Right coronary artery fistula to the coronary sinus and right atrium associated with giant right coronary enlargement detected by transthoracic echocardiography. Eur J Echocardiogr 2011;12:E22.

8. Iwata S, Inano C, Ozaki M. Perpendicular and turbulent flow after aortic valve replacement: paravalvular or transvalvular leakage? - a case report. J Cardiothorac Surg 2020;15:19.

9. Weber CA, Prasad R, Ballmoos MW, et al. New regurgitation after aortic valve replacement with a stented 
bovine pericardial prosthesis: is the leak paravalvular or non-paravalvular? J Cardiothorac Vasc Anesth 2014;28:857-9.

10. Matteucci M, Ferrarese S, Cantore C, et al. Early Aortic Paravalvular Leak After Conventional Cardiac Valve Surgery: A Single-Center Experience. Ann Thorac Surg 2020;109:517-25.

11. O'Rourke DJ, Palac RT, Malenka DJ, et al. Outcome of mild periprosthetic regurgitation detected by intraoperative transesophageal echocardiography. J Am Coll Cardiol 2001;38:163-6.

12. Ionescu A, Fraser AG, Butchart EG. Prevalence and clinical significance of incidental paraprosthetic valvar regurgitation: a prospective study using transoesophageal echocardiography. Heart 2003;89:1316-21.

13. Unzué L, García E, Díaz-Antón B, et al. Percutaneous Closure of Mitral Paravalvular Leaks Generating Refractory Heart Failure in the Immediate Postoperative Period. J Invasive Cardiol 2019;31:E259-60.

14. Alkhouli M, Sarraf M, Maor E, et al. Techniques and Outcomes of Percutaneous Aortic Paravalvular Leak Closure. JACC Cardiovasc Interv 2016;9:2416-26.

15. Nietlispach F, Maisano F, Sorajja P, et al. Percutaneous

Cite this article as: Zhou H, Chen H, Tan L, Li Y. Perpendicular and turbulent flow in acute aortic paravalvular leak: case report. Ann Palliat Med 2021;10(8):9318-9323. doi: 10.21037/apm-21-698 paravalvular leak closure: chasing the chameleon. Eur Heart J 2016;37:3495-502.

16. Smolka G, Pysz P, Wojakowski W, et al. Clinical manifestations of heart failure abate with transcatheter aortic paravalvular leak closure using Amplatzer vascular plug II and III devices. J Invasive Cardiol 2013;25:226-31.

17. Onorato EM, Muratori M, Smolka G, et al. Midterm procedural and clinical outcomes of percutaneous paravalvular leak closure with the Occlutech Paravalvular Leak Device. EuroIntervention 2020;15:1251-9.

18. Smolka G, Pysz P, Jasiński M, et al. Multiplug paravalvular leak closure using Amplatzer Vascular Plugs III: A prospective registry. Catheter Cardiovasc Interv 2016;87:478-87.

19. Saritas T, Kaya MG, Lam YY, et al. A comparative study of Cardi-O-Fix septal occluder versus Amplatzer septal occluder in percutaneous closure of secundum atrial septal defects. Catheter Cardiovasc Interv 2013;82:116-21.

20. Celebi A, Demir IH, Saritaş T, et al. Cardi-O-Fix duct occluder versus Amplatzer duct occluder for closure of patent ductus arteriosus. Catheter Cardiovasc Interv 2013;82:961-7. 